\title{
Flexible Scheme for Measuring Chromatic Dispersion Based on Interference of Frequency Tones
}

\author{
K.R.H. Bottrill, M.A. Ettabib, J.C. Gates, C. Lacava, F. Parmigiani, D.J. Richardson, P. Petropoulos \\ Optoelectronics Research Centre, University of Southampton, UK \\ krhb1g12@soton.ac.uk
}

\begin{abstract}
We propose and demonstrate a flexible new scheme for measuring chromatic dispersion profiles of optical devices. This is achieved by measuring the phase difference between two mutually coherent tones that are mixed together through a modulator.

OCIS codes: (060.2270) Fiber characterization; (120.5710) Refraction; (260.2030) Dispersion.
\end{abstract}

\section{Introduction}

Although there exist several techniques capable of measuring the chromatic dispersion of optical media, the measurement of devices with either a low net dispersion or a short length still presents a challenge [1,2]. The resolution of the conventional differential phase method (RF modulation of an optical carrier, see standard TIA-455175) [3] is limited by the frequency of the modulation. On the other hand, low-coherence spectral interferometry is capable of measuring small values of dispersion (provided that a considerably broadband source is available), but the requirement for path-length matching of the interferometer arms renders it inflexible when the measurement of long-length devices is required. We present here a new method for measuring the chromatic dispersion that overcomes the aforementioned shortcomings by combining the benefits of two different technologies: few-tone highly coherent frequency combs and the capability of applying deterministic phase changes on discrete wavelength channels through a programmable optical filter. The novelty of the scheme comes from the means of measuring these phase shifts, a technique we originally developed in order to measure the phase transfer profile of a phase quantizing phase sensitive amplifier [4]. This technique is simple, robust and very accessible, given the prevalence of the equipment used in its implementation. We demonstrate the potential of the technique by using the same measuring system for both $\mathrm{km}$-long lengths of fiber as well as a cm-long silicon germanium waveguide.

\section{Principle of Operation}

As shown in [4], the phase difference between two mutually coherent optical tones with frequency spacing $\Delta f$ can be measured by applying a phase shift $\sigma$ to one of the tones and mixing them using a Mach-Zehnder modulator (MZM) driven at $\Delta f$. Taking four power measurements of one of the interfering comb lines at four different values of $\sigma,\{0, \pi / 2, \pi, 3 \pi / 2\}$, allows the phase difference of the two lines, $\Phi$, to be extracted according to:

$$
\Phi=\arctan \left(\frac{P_{\sigma=\pi / 2}-P_{\sigma=3 \pi / 2}}{P_{\sigma=0}-P_{\sigma=\pi}}\right)
$$

We propose to use this technique to measure the phase shift between two tones due to chromatic dispersion. Neglecting nonlinearity in the medium under test, the relative phase difference between two tones of radial frequencies $\omega_{1}$ and $\omega_{2}$ caused by a medium of length $L$ and frequency dependent propagation constant $\beta(\omega)$ is given by $\Phi=\left(\beta\left(\omega_{2}\right)-\beta\left(\omega_{1}\right)\right) L+\phi_{\text {off }}$, where $\phi_{\text {off }}$ represents the initial phase difference between the two tones. Dividing $\Phi$ by $\left(\omega_{2}-\omega_{1}\right) L$ can be seen to result in a first order approximation to the group velocity, $\frac{d \beta}{d \omega}$, plus a constant:

$$
\frac{\Phi}{\left(\omega_{2}-\omega_{1}\right) L}=\frac{\beta\left(\omega_{2}\right)-\beta\left(\omega_{1}\right)}{\omega_{2}-\omega_{1}}+\frac{\phi_{o f f}}{L\left(\omega_{2}-\omega_{1}\right)} \approx \frac{d \beta}{d \omega}+\frac{\phi_{o f f}}{L\left(\omega_{2}-\omega_{1}\right)}
$$

We can obtain the chromatic dispersion of the medium by taking the derivative of Eq. 1 with respect to wavelength [5]: $D=\frac{d}{d \lambda}\left(\frac{d \beta}{d \omega}\right)$, which also eliminates the constant $\frac{\phi_{\text {off }}}{L\left(\omega_{2}-\omega_{1}\right)}$.

\section{Experimental Results}

The experimental set-up used is given in Fig. 1, along with typical spectra at various points in the system. First, a tunable continuous wave $(\mathrm{CW})$ laser is modulated by a MZM driven at $30 \mathrm{GHz}$, forming a comb of mutually coherent tones. This comb is then passed through a programmable filter (Finisar Waveshaper), which selects out two tones separated by $60 \mathrm{GHz}$ and applies a phase shift of $\sigma$ on one of them. These tones then pass through a circulator to the device under test (DUT). The DUT is terminated with a Faraday mirror to compensate for any polarization rotation of the tones relative to each other along the DUT, providing consistent results in the case of long, weakly birefringent media such as non-polarization maintaining fibers (note that this means the system is exposed to twice 


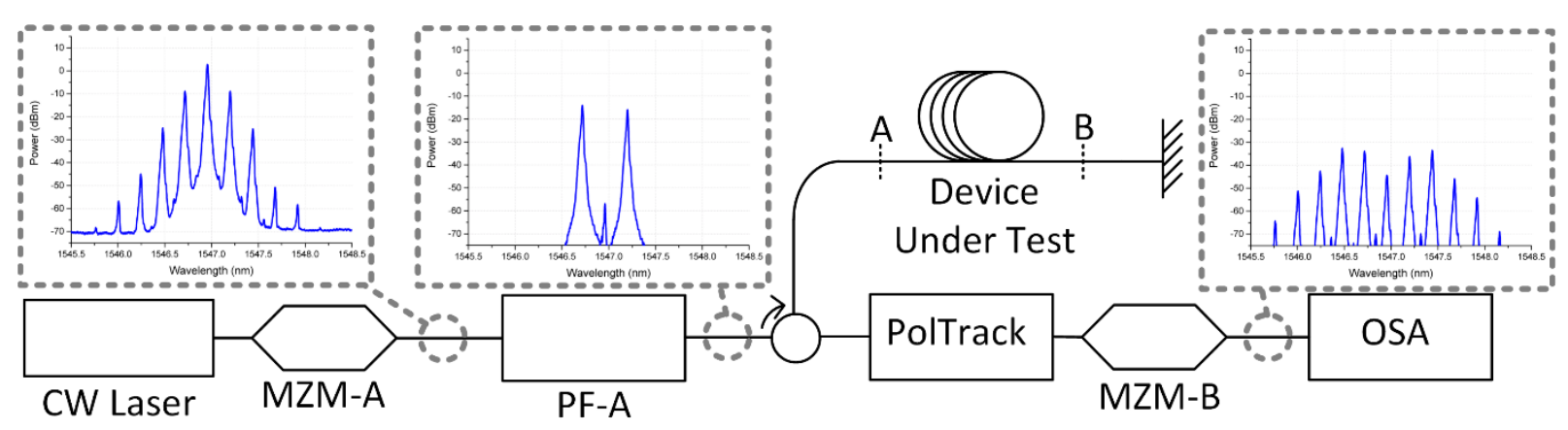

Figure 1: Experimental set-up of the scheme with associated spectra at various locations in the system.

the dispersion of the DUT). The counter-propagating wave exits through the third port of the circulator, where it first enters a polarization tracker, ensuring that it can be launched appropriately into the following MZM. This second MZM, driven at exactly the same frequency as the first, causes the two tones to interfere. The power of the tone formed at the location of the original $\mathrm{CW}$ carrier is then measured using an optical spectrum analyzer (OSA) for the four different values of $\sigma$ necessary to calculate the relative phase between the two lines, $\Phi$.

By tuning the original CW carrier, it was possible to trace $\Phi(\lambda)$ for $1528 \mathrm{~nm} \leq \lambda \leq 1565 \mathrm{~nm}$ (the bandwidth of the C-band programmable filter used), the step size of $\lambda$ being chosen to prevent changes in $\Phi(\lambda)$ greater than $2 \pi$ between two successive wavelengths. Note that for calibration, a background measurement of $\Phi(\lambda)$ without the DUT needs to be subtracted from the measured phase profile. To illustrate this, Figs. 2-a and -b provide plots of $\Phi(\lambda)$ with and without $200 \mathrm{~m}$ of unstrained SPINE HNLF (a product of OFS) in the system, respectively. Experimental data are plotted with orange triangles and quadratic fits are plotted with blue lines. Subtracting the fitting data shown in Fig. 2-b from that in Fig. 2-a results in the genuine trace of $\Phi(\lambda)$ for the SPINE HNLF given in Fig. 2-c. From this stage, dispersion can be obtained simply by differentiating $\Phi(\lambda)$ with respect to $\lambda$ and dividing the result by $L\left(\omega_{2}-\omega_{1}\right)=0.2 \mathrm{~km} \times\left(2 \pi \times 60 \times 10^{-9} \mathrm{GHz}\right)$, as discussed above, resulting in the dispersion curve provided in Fig. 2-d. Also plotted in Fig. 2-d is a measurement on the same fiber made using the differential phase method (see standard TIA-455-175), which show very good agreement with one another.

Measurements we also made upon $1 \mathrm{~km}$ of Corning SMF-28, which is a well known and well specified fiber. Corning provide the following analytical equation to approximate the chromatic dispersion of SMF-28: $D_{28}(\lambda) \approx$ $\frac{S_{0}}{4}\left(\lambda-\lambda_{0}^{4} / \lambda^{3}\right)$, where $S_{0} \leq 0.092 \mathrm{ps} \mathrm{nm}^{-1} \mathrm{~km}^{-1}$ and $1302 \mathrm{~nm} \leq \lambda_{0} \leq 1322 \mathrm{~nm}$. Fig. 2 -c provides the chromatic dispersion plot obtained using our technique (solid orange line), which gives $D=17.5 \mathrm{ps} \mathrm{nm}^{-1} \mathrm{~km}^{-1}$ and $D^{\prime}=$ $0.060 \mathrm{ps} \mathrm{nm}^{-2} \mathrm{~km}^{-1}$, along with $D_{28}(\lambda)$ plotted for $\lambda_{0}=1308 \mathrm{~nm}$ (dashed blue line). A very good agreement can be seen between the analytical approximation and the results obtained through the experiment, confirming the functioning of the scheme.

To demonstrate the scheme's ability to measure low net values of dispersion, we used our dispersion measurement system to characterize the dispersion of three different lengths of single-mode fiber patchcord (of lengths $1 \mathrm{~m}, 3.1 \mathrm{~m}$ and $6.1 \mathrm{~m}$ ) as well as three different combinations of these patchcords by connecting them using uniters. Given the longitudinal non-uniformity of dispersion in typical SMF[6], as well as the short lengths of SMF used in this demonstration (on the order of metres), we cannot expect dispersion measurements upon these patchcords to replicate the nominal values of SMF-28, nor even for them to be in direct agreement with one another. Six different combinations of fiber were measured using our scheme which we enumerate in Table 1. Figure 2-d provides a plot of the net chromatic dispersion estimates obtained from the direct $10.2 \mathrm{~m}$ measurement, M6, as well as those obtained by adding the measured dispersion of the single patchcords such that their combination gives the same length of $10.2 \mathrm{~m}$, i.e. combinations $\mathrm{M} 1+\mathrm{M} 2+\mathrm{M} 3, \mathrm{M} 1+\mathrm{M} 5$ and $\mathrm{M} 2+\mathrm{M} 4$. Ideally, all these curves should be equal and any deviation will give an estimate of the measurement error. All of the results can be seen to be in good agreement with each other, proving the consistency of the measurement.

\begin{tabular}{|c|c|c|c|c|c|c|}
\hline Name & M1 & M2 & M3 & M4 & M5 & M6 \\
\hline Combination & $1 \mathrm{~m}$ & $3.1 \mathrm{~m}$ & $6.1 \mathrm{~m}$ & $1+6.1=7.1 \mathrm{~m}$ & $3.1+6.1=9.2 \mathrm{~m}$ & $1+3.1+6.1=10.2 \mathrm{~m}$ \\
\hline
\end{tabular}

The fine measuring resolution of the technique (which is better than $0.03 \mathrm{ps} \mathrm{nm}^{-1}$, the dispersion of M1) allows us to measure directly the dispersion of waveguide devices exhibiting a low net dispersion, and to demonstrate this, we measured the dispersion of a $2 \mathrm{~cm}$ long $\mathrm{Si}_{0.8} \mathrm{Ge}_{0.2}$ low birefringence waveguide (shown in Fig. 3-a), resulting in the curve shown in Figure 3-b. (Note that to take this measurement, it was necessary to remove the Faraday mirror and operate the scheme in a single pass mode, due to the strong back reflections from the waveguide facet.) 

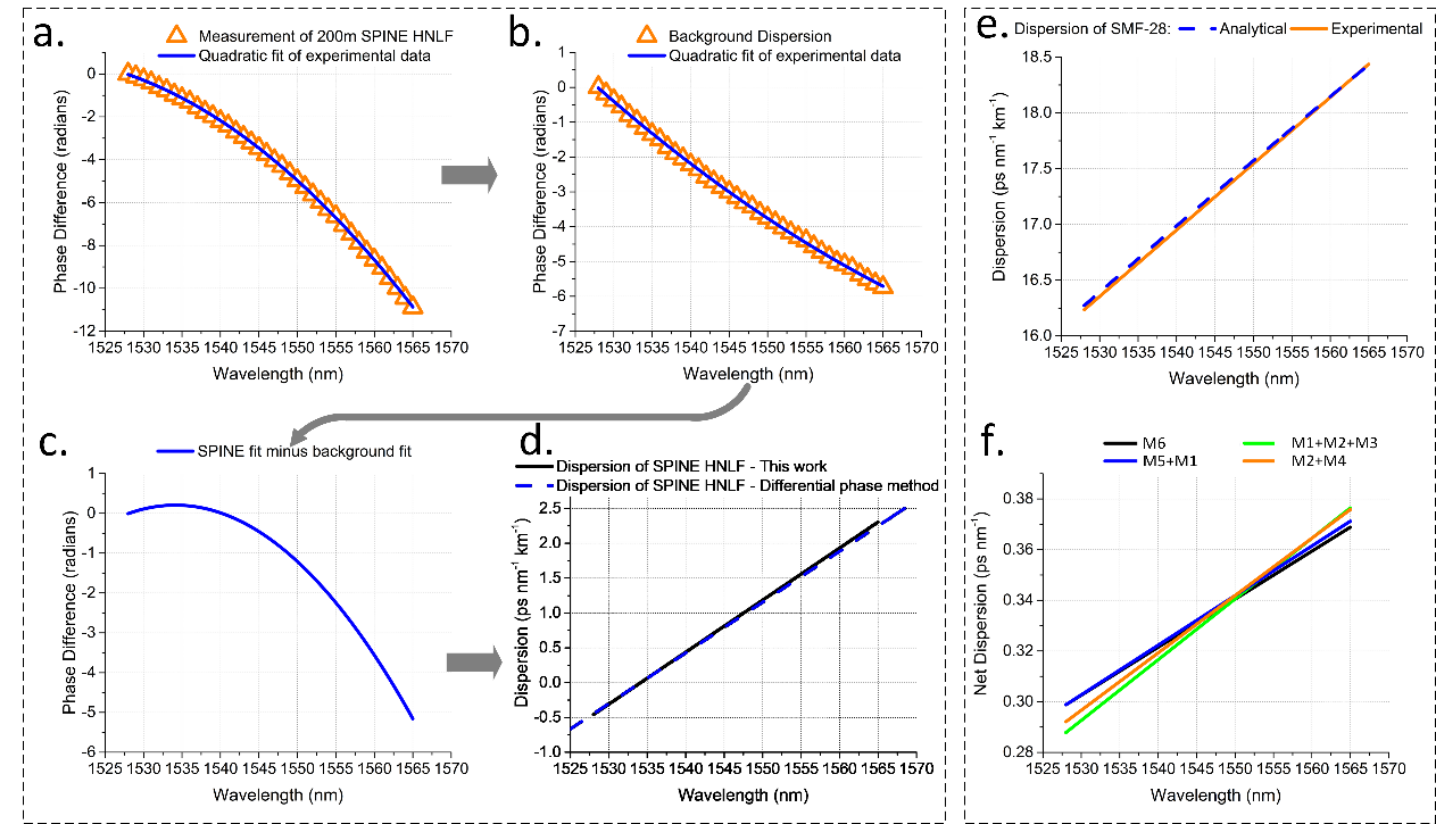

Figure 2: a-d: Procedure to measure dispersion. e: Dispersion of $1 \mathrm{~km}$ of SMF-28. f: Dispersion of patchcord combinations.
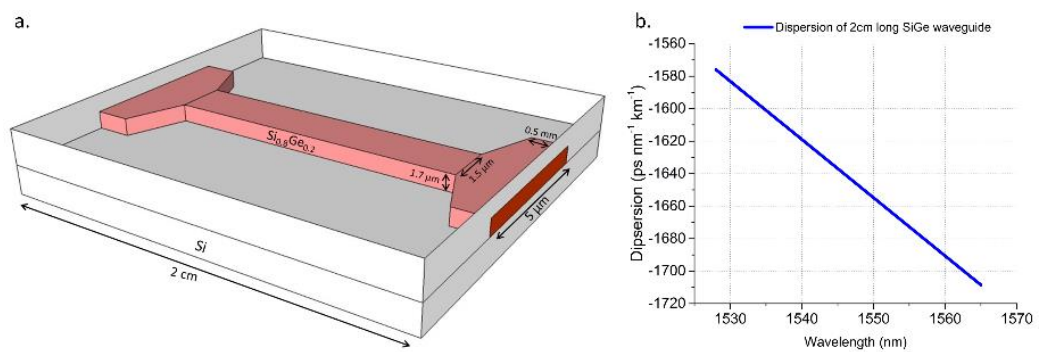

Figure 3: a. Diagram of the $2 \mathrm{~cm}$ long $\mathrm{Si}_{0.8} \mathrm{Ge}_{0.2}$ waveguide. b. Measured dispersion of the waveguide.

\section{Discussion and Conclusion}

We have described and demonstrated a novel system capable of measuring low levels of net chromatic dispersion using only two Mach-Zehnder modulators, a programmable filter and an OSA. The scheme is notable for being able to measure net chromatic dispersions lower than $0.03 \mathrm{ps} \mathrm{nm}^{-1}$, a limit which can be improved by increasing the tone separation. Exploiting this effect allows the scheme to measure a great range of dispersions, as well as offering the possibility of high dynamic range using an adaptive scheme. This ability to measure low values of net chromatic dispersion was demonstrated upon a $2 \mathrm{~cm}$ long Silicon Germanium waveguide. Additionally, as the two probe tones are co-propagating, they remain mutually coherent over very long lengths, obviating the need for path length matching or lasers with long coherence lengths and eliminating concerns for mechanical stability of the system.

\section{Acknowledgments}

The work is part of the EPSRC Photonics Hyperhighway Programme Grant. F. Parmigiani is a Royal Academy of Engineering/EPSRC Research Fellow.

\section{References}

[1] P. Merrit, R. P. Tatam, and D.A. Jackson, "Interferometric chromatic dispersion measurements on short lengths of Monomode optical fiber," J. Lightwave Technol. 7, 703-716 (1989).

[2] Vicente Durán, Peter A. Andrekson, and Víctor Torres-Company, "Electro-optic dual-comb interferometry over $40 \mathrm{~nm}$ bandwidth," Opt. Lett. 41, 4190-4193 (2016)

[3] B. Costa, D. Mazzoni, M. Puleo, E, Vezzoni, “ Phase Shift Technique for the measurement of Chromatic Dispersion in Optical Fibers using LED's", IEEE Transactions on Microwave Theory and Techniques, vol. 82, Issue 10, p. 1497-1503 (1982).

[4] K. R. H. Bottrill, G. D. Hesketh, F. Parmigiani, P. Horak, D. J. Richardson and P. Petropoulos, "Suppression of Gain Variation in a PSABased Phase Regenerator Using an Additional Harmonic," in Photonics Technology Letters, vol. 26, no. 20, pp. 2074-2077, Oct.15, 15 2014.

[5] Govind Agrawal. Nonlinear Fiber Optics, Fifth Edition (Optics and Photonics). Academic Press, 2012.

[6] E. Myslivets, N. Alic and S. Radic, "Spatially Resolved Measurement in Waveguides With Arbitrary Chromatic Dispersion," in IEEE

Photonics Technology Letters, vol. 20, no. 21, pp. 1793-1795, Nov.1, 2008. 